\title{
Chronic localised encephalitis (Rasmussen's) in an adult with epilepsia partialis continua
}

\author{
FRANCOISE GRAY,* MICHEL SERDARU, HENRY BARON,§ \\ CATHERINE DAUMAS-DUPORT, $\uparrow$ PHILIPPE LORON, $\dagger$ BRUNO SAURON, $\|$ \\ JACQUES POIRIER*
}

\begin{abstract}
From Département de Pathologie (Neuropathologie), * Service de Réanimation Médicale, $†$ Hopital Henri Mondor Créteil, Clinique de Neurologie et de Neuropsychologie $\ddagger$ Laboratoire de Neurovirologie,§ Clinique des Maladies du Systême Nerveux, || Hopital de la Salpetrière, Laboratoire d'Anatomie Pathologique, đ Hopital Sainte Anne, Paris, France
\end{abstract}

SUMMARY A 29 year old male presented with epileptic fits, progressive left sided focal seizures and epilepsia partialis continua, increasing left hemiparesis and mental slowing. Death occurred 2 years after the onset of the illness. Lesions were limited to the right cerebral hemisphere. Hypertrophic astrocytosis was diffuse throughout the gray and white matter but was more severe in the deep cortical layers and $U$ fibres, where it was associated with vacuolar changes and capillary proliferation. Sparse perivascular lymphocytic cuffs, rod shaped microglia and microglial nodules were present. No inclusion bodies were found. These clinico-pathologic features were similar to the cases described by Rasmussen. Only five necropsy cases of this rare disease have been reported previously, all in children. The aetiology is unknown.

Epilepsia partialis continua, first described by Koshewnikow, ${ }^{1}$ is a rare form of focal epilepsy characterised by partial somato-motor status epilepticus due to various lesions of the motor cortex. ${ }^{23} \mathrm{~A}$ rare form of chronic encephalitis was identified by Rasmussen $e t a l^{4}$ on surgical specimens from patients who underwent operation for focal epilepsy. A complete post-mortem examination was carried out only in one infantile case. It showed that the lesion was focal and the condition was named "Chronic localized encephalitis at the origin of focal cerebral seizures". Since this original description only four necropsy cases have been reported to our knowledge. ${ }^{56}$ All of these were children or adolescents.

\section{Case report}

A 29 year old right handed man was admitted to hospital for evaluation of epileptic fits which had started with left facial myoclonus and rapidly extended to the left arm. Clinical

\begin{abstract}
Address for reprint requests: Dr F Gray, Département de Pathologie (Neuropathologie), Hopital Henri Mondor 94010 CRETEIL Cedex, France.
\end{abstract}

Received 18 February 1986 and in revised form 9 May 1986. Accepted 15 May 1986 examination, CSF analysis, EEG and CT scan were normal. He was treated successfully with carbamazepine. Four months later, he developed a choroiditis of the right eye which resolved following steroid-therapy. Six months after onset, the epilepsy recurred in the form of short absences. EEG displayed permanent spikes and slow wave activity over the right fronto-parietal region. A second CT scan was normal. Daily doses of carbamazepine were increased and barbiturates were added without improvement.

Ten months after onset, the patient complained of clumsiness of the left hand and leg and permanent involuntary left-sided movements involving the limbs and the tongue. Neurological examination showed persistent left sided myoclonus, brisk tendon reflexes on the left side and equivocal plantar response. Neuropsychological testing disclosed mild abnormalities on the Wechsler memory test (score 97). CSF examination revealed $56 \mathrm{mg} / \mathrm{dl}$ protein, normal IgG and 7 WBC. The following investigations gave normal results: FBC, ESR, urea nitrogen, serum glucose, $\mathrm{Na}, \mathrm{K}, \mathrm{P}, \mathrm{Ca}$ and visual evoked potentials, investigations for toxoplasmosis, candidiasis, herpes, brucella, sarcoidosis and a liver biopsy. A stereotactic frontal biopsy was performed 16 months after the onset of the illness and will be described below.

Further evolution was characterised by permanent leftsided myoclonic jerks associated with partial or generalised epileptic seizures poorly controlled by high doses of various anticonvulsants, increasing left hemiparesis with hemihypaesthesia, mental slowing, gradual spread of epileptiform activity on serial EEG, and progressive, moderate, right hemispheric atrophy on repeated CT scans. 
The patient became bedridden and died 2 years after onset of the first neurological signs.

\section{Pathological examination}

\section{(1) Biopsy}

The specimens obtained by serial stereotactic frontal biopsy were divided in two parts: one was fixed in $10 \%$ formaldehyde, embedded in paraffin wax and stained by routine histological methods. The other was fixed in $2.5 \%$ glutaraldehyde, post fixed in $1 \%$ osmium tetroxide, embedded in epoxy resin. Thin sections were stained with uranyle acetate and lead citrate for electron microscopy. Histological examination showed proliferation of hypertrophic astrocytes throughout the gray and white matter, most severe in the fifth and sixth cortical layers and $U$ fibres. Sparse perivascular lymphocytic cuffs and proliferation of rod shaped microglia with microglial nodules were observed in the cortex. Microcystic changes were marked in the underlying white matter. No inclusion bodies were found by light or electron microscopy.

(2) Post-mortem examination was carried out 6 hours after death and showed bilateral decubitus ulcers and aspiration bronchopneumonia. The brain was examined after one month of $10 \%$ formalin fixation.

Neuropathological Examination The brain weighed $1600 \mathrm{~g}$ and showed mild right hemispheric atrophy, most severe in the fronto-temporal region, with dilatation of the lateral ventricle more marked in the temporal horn. The right cortical ribbon was thinned in the motor strip and Ammon's horn. The entire junction between cortex and white matter looked reddish-brown on the right side (figs 1,2 ). The left cerebral hemisphere, the cerebellum, brainstem and spinal cord were grossly normal. The arterial supply of the brain was unremarkable.

Microscopic examination Blocks from both cerebral hemispheres, cerebellum, brainstem and spinal cord were embedded in paraplast or in celloidin and stained with haematoxylin and eosin, Loyez stain for myelin, Masson trichrome and Bodian silver impregnation combined with Luxol fast blue. In addition, slides from a representative paraffin block of right parietal cortex were immunostained by the peroxidase-antiperoxidase (PAP) method $^{7}$ for the demonstration of SAF protein. A section of scrapie mouse brain was used as a positive control.
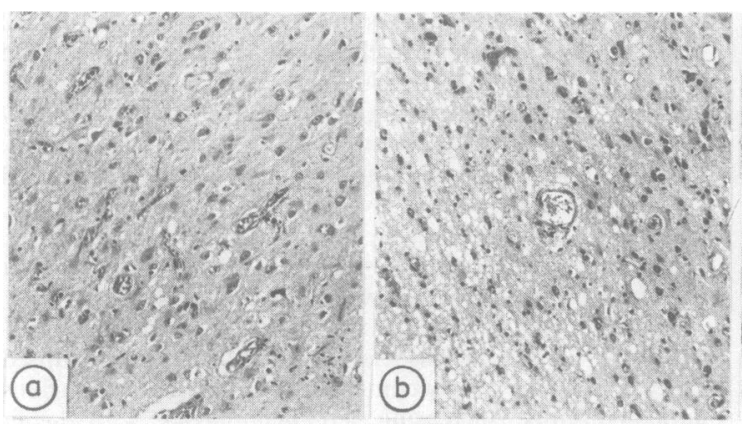

Fig 3 (a, c) fifth and sixth cortical layers, ( $H$ and $E a \times 60, c \times 150$.) capillary proliferation, hypertrophic gliosis, slight spongiosis, relative preservation of the neurons. $(b, d) U$ fibres $H$ and $E(b \times 60, d \times 150$.) marked vacuolation, hypertrophic gliosis and capillary proliferation.

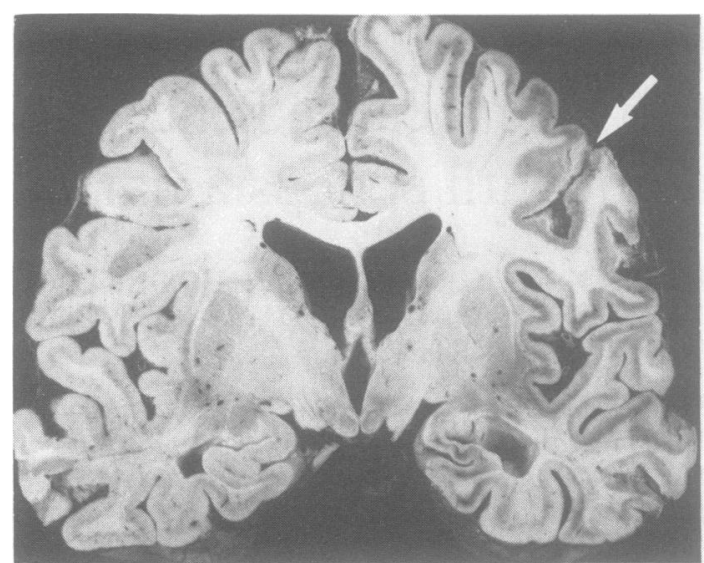

Fig 1 Coronal section of the cerebral hemispheres through the mamillary bodies shows atrophy of the right hemisphere, dilatation of the temporal horn of the right lateral ventricle, necrotic aspect of the opercula (arrow) and discolouration of the deep layers of the cortex.
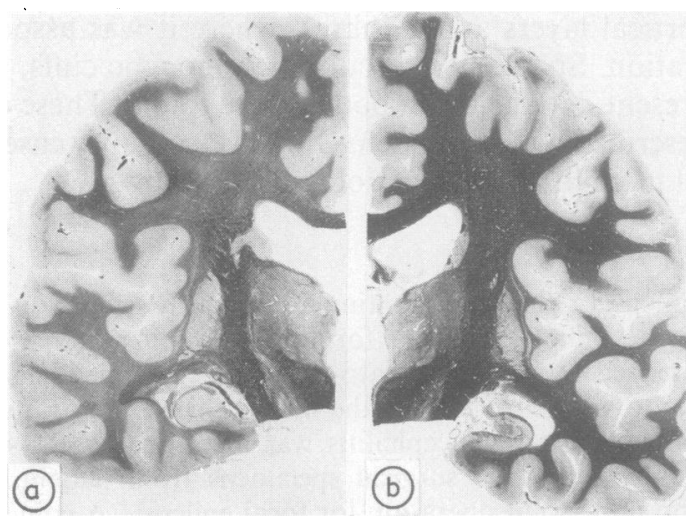

Fig 2 Coronal sections of the cerebral hemispheres through side: cortical atrophy more marked in the precentral gyrus and Ammon's horn, dilatation of the temporal horn of the lateral ventricle.

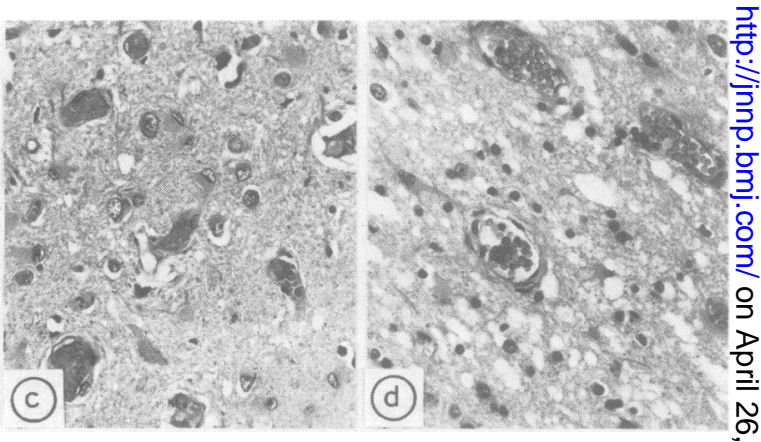

o

กิ

ర్ల the red nuclei. Loyez stain. (a) Left side: normal, (b) Right 

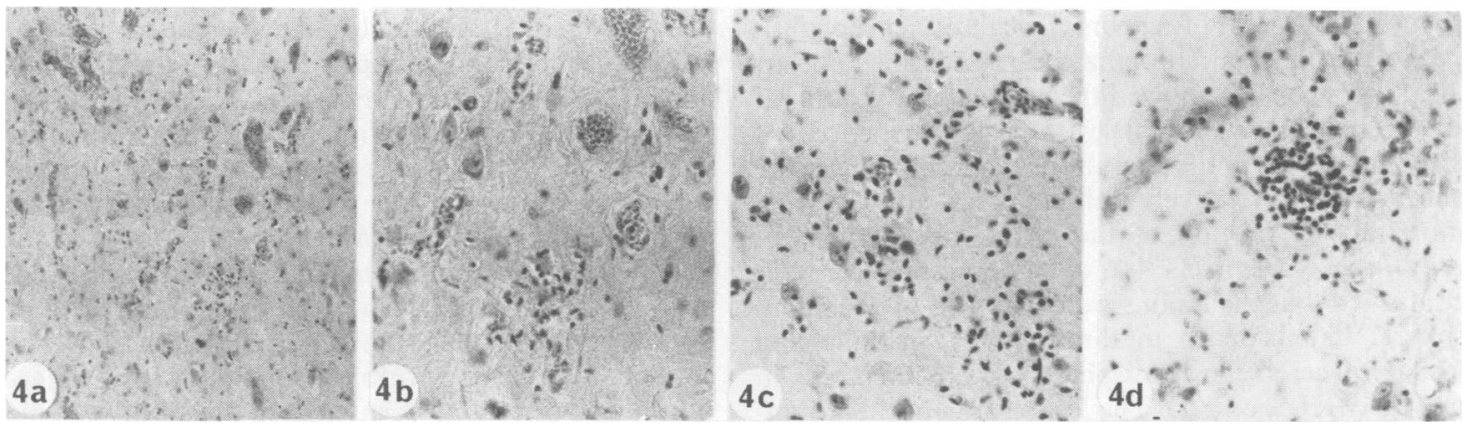

Fig 4 Inflammatory changes in temporal cortex $(H$ and $E) .(a \times 60, b \times 150)$ rod shaped microglia, microglial nodules, relative preservation of the neurons. $(c \times 150)$ microglial proliferation. $(d \times 150)$ perivascular lymphocytic cuff.

Hemispheric lesions were limited to the right side: they involved the cortex predominantly and were severe in the precentral gyrus, especially in the pars opercularis where nerve cells were reduced and microvacuoles occupied the whole thickness of the cortex except the first layer. Ammon's horn showed mild neuronal loss, capillary proliferation and gliosis with Rosenthal fibres. The remaining cortex was better preserved: neurons were usually spared, vacuolation was less severe and was confined to the fifth and sixth layers with extension to the subcortical white matter. In these latter areas it was associated with massive hypertrophic gliosis and capillary proliferation (fig 3 ). Sparse rod shaped microglia, microglial nodules and lymphocytic perivascular cuffing were present throughout the whole cortex and $U$ fibres but were more abundant in the better preserved areas (fig 4).

In the deep white matter only mild hypertrophic gliosis and few perivascular cuffs were present. The claustrum showed slight neuronal loss, microvacuolation and massive hypertrophic gliosis. Marked neuronal loss and fibrillary gliosis with Rosenthal fibres were observed in the medial nucleus of the thalamus. The basal ganglia were otherwise unremarkable.

The leptomeninges were thickened and showed increased fibrous tissue; they contained inflammatory mononucleated cells.

In the left hemisphere, the cortex including Ammon's horn, white matter, basal ganglia and leptomeninges were normal.

The brainstem and spinal cord showed myelin loss and atrophy of the cortico-spinal tract. Nerve cell loss and gliosis were present in the right pontine nuclei, left cerebellar hemisphere, left dentate nucleus and ventral lamella of the right inferior olive.

There were neither haemorrhages nor ischaemic neuronal changes. No inclusion bodies could be found at any level of the central nervous system. A section from right parietal cortex immunostained with primary antibody directed against SAF protein disclosed no positive staining structures.

\section{Discussion}

The association in our patient of persistent left sided clonic muscular twitches and focal epileptic seizures affecting one side of the body supports the diagnosis of epilepsia partialis continua. ${ }^{28}$ Since the original description of this syndrome ${ }^{1}$ there has been considerable controversy regarding possible cortical or subcortical mechanisms. Recent investigations which have demonstrated the cortical origin of epilepsia partialis continua ${ }^{23}$ are supported by the morphological findings in the present case. They show the most severe and destructive changes in the right precentral gyrus. We interpret the lesions in the thalamus, brainstem, cerebellum and spinal cord as the consequence of this motor strip necrosis. The involvement of the claustrum is unusual and so far unexplained.

In 1958 Rasmussen et $\mathrm{al}^{4}$ first drew attention to the histological features of active encephalitis in surgical specimens from children operated on for intractable focal seizures. In further publications ${ }^{9-12}$ Rasmussen stressed the distinctive clinical and histological features of what he called "chronic localised encephalitis at the origin of focal cerebral seizures." Since then a few more case reports of such "smouldering encephalitis" have appeared" 613-15 some without detailed clinical or neuropathological description. ${ }^{16-18}$

The clinical data in our observation are very similar to those previously reported as chronic localised encephalitis: they include medically refractory focal seizures, often associated with episodes of epilepsia partialis continua, slowly progressive neurological deterioration with hemiparesis and mental slowing, normal CSF or transitory protein elevation with variable increase of WBC, localised epileptiform activity increasing in severity and extent on serial EEGs, progressive atrophy of one hemisphere on repeated pneumoencephalograms or CT scans, progressive course over a period of years with spontaneous stabilization in most cases; in the most severe forms, death occurring in the first two years. However the adult onset of the disease in the present case is atypical; in a series of 27 patients, ${ }^{9}$ only one became ill at age 31; all the remaining patients had their first seizures prior to age 15 years. 
In most cases of chronic localised encephalitis pathological examination has been performed on surgical specimens. It showed the association of destructive changes with little or no evidence of inflammation, and alterations typical of active encephalitis in relatively well preserved areas. A post mortem examination of the brain was carried out only in six cases (refs 4-6 and the present case). It confirmed that the lesions were confined to one hemisphere. Extension to midbrain, pons, medulla and ipsilateral cerebellar hemisphere was observed only in one case (ref 5, case 5). The lesions involved predominantly the fronto-temporal cortex and were more marked in the fifth and sixth layers extending to the $U$ fibres. A concentric pattern was obvious in our case: the fronto-temporal and to a lesser extent parietal areas surrounding the necrotic pars opercularis of the precentral gyrus, contained severe gliosis and vacuolation. These latter changes were milder in peripheral regions which showed more marked inflammatory alterations. This supports the hypothesis of a centrifugal spread of the disease which was already suspected on clinical grounds and serial histological examinations. ${ }^{510}$

The aetiology of the disease remains undetermined. Although a viral encephalitis is suggested on histological grounds, none of the patients had developed an obvious encephalitic episode and the CSF was usually normal. Nevertheless an inflammatory episode of some sort is frequently found at, or shortly before, the onset of seizures. It is of interest that our patient developed a choroiditis on the same side as the hemispheric lesions. In addition, repeated lumbar punctures showed transitory protein elevation and/or WBC count abnormalities in many cases. These elements have led Rasmussen ${ }^{9}$ and Gupta $e t$ al $^{5}$ to suggest that some impairment of immune defence mechanisms, genetic peculiarities, or viral mutation could account for the chronic course of a virus induced encephalitis as in subacute sclerosing pancencephalitis. It is of interest that one case (ref 5 , case 4) suffered from the familial syndrome of multiple endocrinopathies, chronic mucocutaneous candidiasis, and impaired cellular immunity. In addition one of the only children with smouldering encephalitis from whom virus isolation was successful, suffered from agammaglobulinaemia. ${ }^{19}$ However, except for the Russian patients with epilepsia partialis continua and tick-borne encephalitis, ${ }^{20-21}$ viral studies on patients' serum CSF, or brain tissue have been negative, ${ }^{51016}$ electron microscopy has failed to show viral particles except in one case, ${ }^{13}$ and immunostaining for viral antigens has been negative. ${ }^{6}$

In a series of 23 patients with Koshewnikow's syndrome Bancaud et $a^{3}$ separated two groups on clinical and electroencephalographic grounds. The first group presented with well defined, limited, cortical lesions. The second group with early onset, clinical and EEG evidence of progressive spread of the lesions, without any obvious cause, was consistent with chronic localised encephalitis. In the latter group the authors suggested the hypothesis of a slow virus infection. No pathological examination was available. In the present case, inflammatory changes were sparse; the prominent histologic features consisted of gliosis and vacuolar changes, which, though not specific, may be compatible with a slow virus infection. For this reason, we attempted to identify scrapie associated fibrils in a representative section of cerebral cortex. These are abnormal amyloid-like fibrils which are considered a specific marker for all the transmissible spongiform encephalopathies due to slow, unconventional viruses. ${ }^{23}$ The recent production of antibody to this structure ${ }^{24}$ has made it possible to demonstrate SAF in brain section by means of a PAP procedure. In the present case this immunostaining was negative. While a positive reaction would have provided strong evidence of an unconventional slow virus infection, a negative reaction does not necessarily rule it out. A piece of brain tissue from our case has been deformalised to attempt an animal transmission study.

The authors thank Doctor H Diringer for providing primary anti SAF antibody, Doctor H Fraser for pro viding scrapie mouse brain, Professors $Y$ Agid, Borie, P Castaigne, HP Cathala, F Chain, JP Chod kiewicz, F Lhermitte, $M$ Rapin and C Vedrenne, ane Doctors F Cathala and C Sicard for permission to publish clinical and paraclinical data, M Favolini for histological preparations and $\mathrm{P}$ Miele for photography.

\section{References}

1 Koshewnikow L. Eine besondere Form von kortikaler Epileptie. Neurol Zblatt 1895;14:47-48.

2 Thomas JE, Reagan TJ, Klass DW. Epileptia partialis continua. A review of 32 cases. Arch Neurol 1977;34:266-75.

3 Bancaud J, Bonis A, Trottier S, Talairaci J, Dulac O. L'epilepsie partielle continue: syndrome et maladie. Rev Neurol (Paris) 1982;138:803-14.

4 Rasmussen T, Olszewsky J, Lloyd Smith D. Focal seizures due to chronic localized encephalitis. Neurology 1958;8:435-45.

5 Gupta PC, Rapin I, Horoupian DS, Roy S, Llena JF Tandon PS. Smoldering encephalitis in children. Neuropediatrics 1984;15:191-7.

6 Mizuno Y, Chou SM, Estes ML, Erenberg G, Cruse RP, Rothner AD. Chronic localized encephalitis (Rasmussen's) with focal epilepsy revisited. (Abstract) $J$ Neuropathol Exp Neurol 1985;44:351. 
7 Sternberger LA, Hardy PH, Cuculis JJ, Meyer HG. The unlabeled antibody enzyme method for immunohistochemistry. Preparation and properties of soluble antigen-antibody complex (horseradish peroxydaseanti-horseradishperoxydase) and its use in identification of spirochetes. $J$ Histochem Cytochem 1970;18:315-33.

8 Juul Jansen P, Denny Brown D. Epileptia partialis continua. A clinical electroencephalographic and neuropathological study of nine cases. Arch Neurol 1968;15:563-78.

9 Rasmussen T. Further observations on the syndrome of chronic encephalitis and epilepsy. Appl Neurophysiol 1978;41:1-12.

10 Rasmussen T, McCann W. Clinical studies of patients with focal epilepsy due to "Chronic Encephalitis" Trans Am Neurol Assoc 1968;93:89-94.

11 Aguilar MJ, Rasmussen T. Role of encephalitis in pathogenesis of epilepsy. Arch Neurol 1960;2:663-76.

12 Rasmussen T, Gossmann H. Epilepsy due to gross destructive brain lesions. Results of surgical therapy. Neurology 1963;13:659-69.

13 Friedman H, Ch'ien L, Parham D. Virus in brain of child with hemiplegia, hemiconvulsions and epilepsy. Lancet 1977;2:666.

14 Gupta PC, Roy S, Tandon PN. Progressive epilepsy due to chronic persistent encephalitis, report of four cases. J Neurol Sci 1974;22:105-20.

15 Lou 'HC, Brandt S, Bruhn P. Progressive aphasia and epilepsy with a self limited course. In: Penry JK, ed. Epilepsy, The Eighth International Symposium. New York: Raven Press, 1977:295.

16 Johnson RT, Herndon RM. Virologic studies of multiple sclerosis and other chronic and relapsing neurological diseases. Prog Med Virol 1974;18:214-28.

17 Goldring S. A method for surgical management of focal epilepsy especially as it relates to children. $J$ Neurosurg 1978;49:344-56.

18 Rocca U, Niedermeyer E. Severe forms of focal motor seizures disorders in childhood. Electroencephalogr Clin Neurophysiol 1980;49:107.

19 Wilfert CM, Buckely RH, Mohanakumar T, Griffith JD, Katz S, Whisnant JK, Eggleston PA, Moore M, Treadwell E, Oxman MN, Roen FS. 1 Persistent and focal central nervous system virus ECHO infections in patients with agammaglobulinemia. $N$ Engl $J$ Med 1977;286:1485.

20 Asher DM. Persistent tick-borne encephalitis infection in man and monkeys: Relation to chronic neurologic disease. In Kurstak E, ed. Arctic and Tropical Arboviruses, Proceedings of the second international symposium on Arctic arboviruses. New York: Academic Press, 1979:179.

21 Brody JA. Chronic sequelae of tick-borne encephalitis and Vilyuisk encephalitis. In: Gajdusek DC, Gibbs $\mathrm{Jr}$ CJ, Alpers $\mathrm{M}$, eds. Slow Latent and Temperate Virus Infections. NINDB monograph No 2. Washington: US Government Printing Office, 1965:111-44.

22 Ilienko VI, Komandenko NI, Platonov VG, Prozorova IN, Panov AG. Pathogenetic study on chronic forms of tick-borne encephalitis. Acta Virol 1974;18:341.

23 Merz PA, Rohwer RG, Kascsak R, Wisniewski HM, Somerville RA, Gibbs CJ Jr, Gajdusek DC. Infectionspecific particle from the unconventional slow virus diseases. Science 1984;225:437-40.

24 Diringer H, Rahm HC, Bode L. Antibodies to proteins of scrapie-associated fibrils. Lancet 1984;ii:345. 